\title{
Lack of an association between SNPs within the cholinergic receptor genes and smoking behavior in a Czech post-MONICA study
}

\author{
Jaroslav A. Hubacek ${ }^{1}$, Vera Lanska ${ }^{2}$ and Vera Adamkova ${ }^{3}$ \\ ${ }^{1}$ Center for Experimental Medicine, Institute for Clinical and Experimental Medicine, \\ Prague, Czech Republic. \\ ${ }^{2}$ Statistical Unit, Institute for Clinical and Experimental Medicine, Prague, Czech Republic. \\ ${ }^{3}$ Department of Preventive Cardiology, Institute for Clinical and Experimental Medicine, \\ Prague, Czech Republic.
}

\begin{abstract}
Smoking has a significant heritable component of approximately 30-60\%. Recent genome wide association studies have identified single nucleotide polymorphisms (SNPs) within the nicotinic cholinergic receptor subunits 3 (rs578776), 5 (rs16969968) and $\beta 3$ (rs6474412), which are associated with nicotine dependence in Western European populations. To analyze the association in a Czech population, we genotyped 1,191 males and 1,368 females (post-MONICA study). The WHO protocol was used to examine smoking status and the number of cigarettes smoked per day. There were $32.1 \%$ current and $27.6 \%$ past smokers among the males and $22.5 \%$ current and $13.8 \%$ past smokers among the females. We have not confirmed the original results: the SNPs rs16969968 $(p=0.07)$, rs578776 $(p=0.16)$ and $r s 6474412(p=0.76)$ were not associated with smoking status (never-smokers vs. ever-smokers) in the entire population, if a codominant model of analysis was used. This result was valid for both the male and female subpopulations if analyzed separately and adjusted for age. Finally, in ever-smokers, the number of cigarettes smoked per day was also independent of different genotypes, regardless of which polymorphism (and gender) was analyzed (the lowest $\mathrm{p}$ value was 0.49 ). The association between the cholinergic receptors-nicotinic subunits $(-3,-5$ and -133$)$, and smoking behavior may be population-dependent.
\end{abstract}

Keywords: cholinergic receptors, polymorphism, smoking.

Received: March 14, 2014; Accepted: July 23, 2014.

Cigarette smoking is the most common form of tobacco use and is a major preventable cause of cancer, chronic respiratory diseases and cardiovascular disease. Despite the intensive preventive programs to stop smoking, approximately $30 \%$ of adults continue to smoke worldwide (Zatonski et al., 2012). The environmental influences on tobacco smoking have been described, but twin studies have confirmed that genes also play an important role in smoking initiation, persistence and ability to stop smoking. The heritable component of smoking is estimated to be approximately 30-60\% (Li and Burmeistr, 2009, Treur et al., 2014).

To date, many gene candidates, such as D2 dopamine receptor (Munafò et al., 2009), SLC6A3 dopamine transporter (Vandenbergh et al., 2002), BDNF (Suriyaprom et al., 2013) and HTTPLRF (Suriyaprom et al., 2012), have been analyzed. However, the results are inconclusive, often

Send correspondence to Jaroslav A. Hubacek. Center for Experimental Medicine, Institute for Clinical and Experimental Medicine, Videnska 1958/9, 14021 Prague 4, Czech Republic. E-mail: jahb@ikem.cz. due to the relatively low numbers of individuals included in the studies.

Recently, genome-wide association studies (GWAs) have identified single nucleotide polymorphisms (SNPs) within or near the subunits of the cholinergic receptors nicotinic, 3 (OMIM acc. No. 118503, rs578776), 5 (OMIM acc. No. 118505, rs16969968), which is located within the CHRNA5/A3/B4 gene cluster at $15 \mathrm{q} 25$, and $\beta 3$ (OMIM acc. No. 118508 , rs6474412), which is located at $8 \mathrm{p} 11$. These SNPs are associated with nicotine dependence and the number of cigarettes smoked per day in Western European populations (Bierut 2009, Thorgeirsson et al., 2010).

Nicotinic cholinergic receptors represent a large group of conserved proteins that code for ligand-gated ion channels. These proteins mediate quick signal transmission at synapses, and because they bind nicotine, they are in a pathway responsible for the physiological responses to smoking. Also the results of mouse knock out models demonstrated the importance of these receptors in nicotine transport (Ware et al., 2012). 
Based on the previously published results (Bierut, 2009, Thorgeirsson et al., 2010, Ware et al., 2012), we supposed that the cholinergic receptor SNPs are associated with smoking behavior (ever- vs. never-smoking, past smoking, number of cigarettes smoked per day in smokers) in a Czech central European population.

Variants within the cholinergic receptors were analyzed in adults aged 34-73 years at the time of examination in 2000/2001, which included 1,191 males and 1,368 females (post-MONItoring of CArdiovascular disease study) (Cifkova et al., 2010). Written informed consent was acquired from all individuals, and the study was approved by the institutional Ethics Committee and was conducted in agreement with the Helsinki Declaration of 1975.

The WHO protocol (Tunstall-Pedoe et al., 2003) was used to examine smoking status and the number of cigarettes smoked per day in 1997/1998, 2000/2001 and 2007/2008. Information about the current smoking status (current-, past- and never- smoker) and the number of cigarettes smoked per day was collected via self-completed (under supervision of trained nurse) questionnaires (Cifkova et al., 2010). Never-smokers were self-reported individuals who had consumed fewer than five packages during their lifetime. Further forms of tobacco use (chewing and snuffing) were not self-reported within the population. Complete information was available from the first two examinations (conducted in 1997/1978 and 2000/2001) and from $84.4 \%$ of individuals examined in 2007/2008.

DNA was isolated using a slightly modified version of the method of Miller et al. (1988). Individual genotypes were analyzed by PCR-RFLP using PCR chemicals and restriction enzymes from Fermentas International Inc. (Burlington, Ontario, Canada). PCR incubations were performed using MJ Research DYAD Disciple PCR cyclers. Oligonucleotide sequences (some primers were mismatched to create new restriction sites) and further details are summarized in Table 1. Restriction fragments were separated via horizontal gel electrophoresis on a $10 \%$ polyacrylamide gel using the MADGE platform (Day et al., 1996).

Deviations from Hardy Weinberg equilibrium were tested using an online calculator (Court 2005-2008). The differences in the genotype frequencies between the groups were assessed using a Chi-square test $(3 \times 3$ or $3 \times 2$ tables) in dominant, codominant and recessive models for both of the years of examination. Table 2 presents the $p$ values for all three unadjusted models at first examination. The number of cigarettes smoked per day in association with individual genotypes was analyzed by ANOVA in the dominant, codominant and recessive models for all years of examination. Table 3 shows the numbers and $p$ values obtained for the codominant model in the second examination. Due to the large differences between males and females in the number of cigarettes smoked per day, the entire population was not analyzed. The results presented in detail are from the examination conducted in 2000/2001 because there were no significant differences among the three examinations.

There were $32.1 \%$ current and $27.6 \%$ past smokers in males and $22.5 \%$ current and $13.8 \%$ past smokers in females in 2000/2001. The mean number of cigarettes smoked per day was $15.7 \pm 8.7$ in males $(\min =1, \max =50)$ and $11.3 \pm 6.4(\min =1, \max =61)$ in females

The call rate was over $93.9 \%$ for each analyzed SNP, and the call rates were similar in smokers and non- or past smokers. The genotype distributions of all three analyzed polymorphisms were within Hardy-Weinberg equilibrium ( $\mathrm{p}=0.19$ for $\mathrm{rs} 16969968, \mathrm{p}=0.44$ for $\mathrm{rs} 578776$ and $p=0.79$ for rs6474412) and did not differ significantly between males and females. Minor allele frequencies were similar to the frequencies found in other Caucasian populations (Bierut, 2009, Thorgeirsson et al., 2010).

The SNPs rs $16969968(\mathrm{p}=0.07)$, rs578776 $(\mathrm{p}=0.49)$ and rs6474412 $(\mathrm{p}=0.76)$ were not associated with smoking status (when never-smokers vs. ever-smokers were compared) in the entire population, if the codominant model of analysis was used. Additionally, negative results were observed for both the male and female subpopulations, if analyzed separately (Table 2) and after adjusting for age.

Some borderline differences (non-significant after adjustment for multiple testing) were observed for the rs6474412 SNP only. Here, it appears that CC homozygotes are less likely to be smokers and that, if they do smoke, they have a slightly higher chance to quit smoking.

Finally, in ever-smokers, the number of cigarettes smoked per day was also independent of genotype, regardless of the polymorphism (and gender) analyzed (all pvalues were 0.16 or higher; for details see Table 3 ). However, in agreement with the previous results, a trend for a

Table 1 - Genotyping details for analysis of SNPs of interest.

\begin{tabular}{|c|c|c|c|c|c|}
\hline Polymorphism & Primer sequences & PCR product & Enzyme & Size of restriction fragments (bp) & Allele \\
\hline Rs16969968 & $\begin{array}{l}5 \text { 'atg aag aag tca tgt aga cag gta ctt c } \\
5 \text { 'tac aca tca cag acc tca cgg aca tc }\end{array}$ & $165 \mathrm{bp}$ & TagI & $\begin{array}{c}165 \\
97+68\end{array}$ & $\begin{array}{l}\text { A } \\
\text { G }\end{array}$ \\
\hline Rs6474412 & $\begin{array}{l}5 \text { 'cct ctt ttc ctg tgt cta ttt gat ggc } \\
5 \text { 'ttc acc ctg caa aga tac tca act ctt cac c }\end{array}$ & $134 \mathrm{bp}$ & HaeIII & $\begin{array}{c}133 \\
109+26\end{array}$ & $\begin{array}{l}\mathrm{T} \\
\mathrm{C}\end{array}$ \\
\hline Rs578776 & $\begin{array}{l}5 \text { 'ttc ttt act ggg tct aaa ggg cta tgc c } \\
5 \text { 'atc cac cca gtt tat ggt gta cta ag }\end{array}$ & $167 \mathrm{bp}$ & NlaIII & $\begin{array}{c}167 \\
100+67\end{array}$ & $\begin{array}{l}\mathrm{T} \\
\mathrm{C}\end{array}$ \\
\hline
\end{tabular}




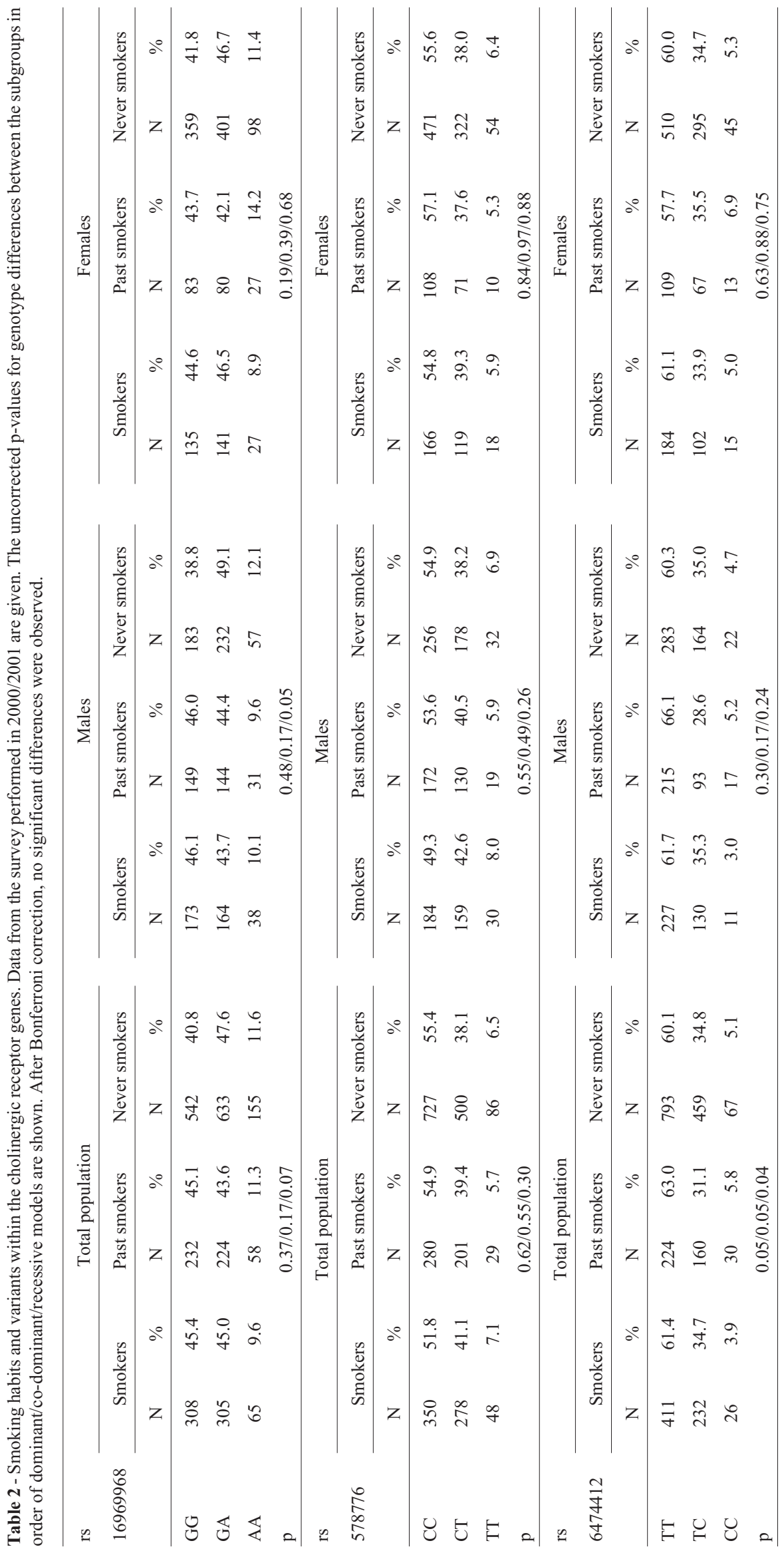


Table 3 - Number of cigarettes smoked per day according the cholinergic receptor gene genotypes. Uncorrected p-values for genotype differences between the subgroups are given (all $\mathrm{p}=$ n.s.). Data from the survey performed in 2000/2001 are presented.

\begin{tabular}{lccccc}
\hline & \multicolumn{2}{c}{ Males } & & \multicolumn{2}{c}{ Females } \\
\cline { 2 - 3 } \cline { 5 - 6 } & $\mathrm{N}$ & N of cigarettes & & $\mathrm{N}$ & N of cigarettes \\
\hline rs16969968 & & & & & \\
GG & 173 & $15.7 \pm 9.1$ & & 135 & $11.4 \pm 6.6$ \\
GA & 164 & $16.2 \pm 8.1$ & & 141 & $10.7 \pm 6.5$ \\
AA & 38 & $18.3 \pm 9.7$ & & 27 & $12.6 \pm 10.9$ \\
p & 0.17 & 0.16 & & & \\
rs578776 & & & & & \\
CC & 184 & $16.7 \pm 8.4$ & & 166 & $11.2 \pm 7.3$ \\
CT & 159 & $15.9 \pm 9.2$ & & 119 & $11.3 \pm 6.9$ \\
TT & 30 & $15.1 \pm 8.6$ & & 18 & $11.6 \pm 6.0$ \\
p & 0.79 & 0.84 & & & \\
rs6474412 & & & & & \\
TT & 227 & $15.9 \pm 8.9$ & & 184 & $11.4 \pm 7.1$ \\
TC & 130 & $16.8 \pm 9.0$ & & 102 & $10.8 \pm 6.5$ \\
CC & 11 & $16.3 \pm 8.0$ & 15 & $11.8 \pm 8.8$ \\
$\mathrm{p}$ & & 0.95 & & & 0.97 \\
\hline
\end{tabular}

higher number of cigarettes smoked per day in the minor AA genotype (rs16969968) carriers was detected.

Similar and negative results were also obtained when the results from examinations conducted in 1997/1998 and 2007/2008 were examined. These results are not shown in detail.

In our study, we did not confirm the original finding that variants within the three nicotinic cholinergic receptors are associated with smoking behavior. This finding was observed in both males and females regardless of the year of examination.

Our data contrast previously published papers that investigated the rs 16969968 polymorphism. This variant represents the amino acid change Asp398Asn $(1192 \mathrm{G}>\mathrm{A})$ at a highly conserved position between species and is, thus, most likely to be of biological significance. Most (Bierut, 2009, Ware et al., 2011, Breetvelt et al., 2012, Conlon and Bewick, 2011, Chen et al., 2012) but not all (Verde et al., 2011) studies have presented an association between the minor Asn (A) allele and higher nicotine dependence (numbers of cigarettes smoked per day or different genotype frequencies between smokers and nonsmokers). An additional study detected that individuals with at least one rs 16969968 allele have a higher risk of being heavy smokers in adulthood if they started to smoke during adolescence (Hartz et al., 2012).

Our data are in agreement with a recent meta-analysis by Ware et al. (2011), who detected a significant effect on the number of cigarettes smoked per day; one risk-allele
(A) was associated with approximately one additional cigarette per day. In our study, we detected a similar effect on the number of cigarettes smoked per day. However, likely due to the large range in the number of cigarettes smoked per day (1-61), the difference was not significant.

The second variant, which was previously described to be associated with nicotine dependency (for a review, see Bierut et al., 2008), rs578776, is located at the same locus as the neighboring gene, but it is not in linkage disequilibrium with rs 16969968 . This variant $(546 \mathrm{C}>\mathrm{T})$ does not change the amino acid, and the mechanism of the potential biological significance is unknown.

The last analyzed SNP (rs6474412) is located at a different region but was also originally associated with the number of cigarettes smoked per day (Thorgeirsson et al., 2010). However in this case, the effect appears to be much lower (one risky $\mathrm{T}$ allele is associated with only an approximately 0.3 cigarette per day increase), and our study did not have sufficient power to detect such a tiny effect.

In our previous work (Hubacek et al., 2012), we were not able to confirm the association between smoking behavior and another GWAs-recognized gene: the FTO gene (Bierut et al., 2007). Due to the geographical location factors like population stratification or founder effect are very unlikely to cause these non-replications. There could be several another reasons for the non-replication(s) on our study.

For example, socioeconomic status may play an important role in smoking behavior because the original GWAs was performed primarily in West European populations. The prevalence of smoking is higher in post-communistic countries, and this fact could mask the real effect of the genes. The fact that most current smokers have or had parents who smoke could reflect not only genetic predisposition but also negative environmental influence.

Secondly, the WHO protocol that we used to select individuals did not necessarily focus on smoking itself, but on smoking as a risk factor of cardiovascular disease. Tests of nicotine dependence that could better reflect nicotine dependence, such as the Fagerström Test for Nicotine Dependence, were not available. Conversely, the number of cigarettes smoked per day represents a simple but representative marker of nicotine dependence (Chen et al., 2012).

Additionally, the numbers of examined individuals within our study are relatively high, and importantly, almost all of the individuals were examined three times within ten years. This frequency decreases the risk of selfreported misinterpretations. Finally, the accuracy of the obtained data is supported by the fact that, in contrast to the alcohol consumption, where clear underestimations, especially in females, are common (Roche and Deehan, 2002), smoking is still not viewed as a "social stigma". The fact that our results were not significant at all three examinations over ten years lowered the risk of false negative results. 
Interestingly, in smokers there was a (not-significant) decrease in the numbers of cigarettes smoked per day over the three examinations. This decrease could reflect the economic situation, rather than genetic predisposition or social factors. With the decreasing number of cigarettes smoked, even slight differences between genotypes and cigarettes smoked per day disappeared.

In summary, the association of SNPs of interest with smoking status (never-, ever-, or past-smokers) was not confirmed in all studies (which could be caused by the low power of some published studies to detect small effects in single variants). Additionally, the commonly analyzed value is the effect of the number of cigarettes smoked per day. Here, the largest studies detected consistent but only very slight effects: one high risk allele is weakly associated with 0.3-1.0 additional cigarette smoked per day. Such a small difference has hardly any clinical significance, especially between the smokers who commonly smoke one or more packages per day. Despite the relatively high number of individuals included, our study may have had insufficient power to detect such small differences.

We observed that the association between the subunits of nicotinic cholinergic receptors $(-3,-5$ and $-\beta 3)$ and smoking behavior may be population-dependent and is likely not valid in the Czech (Slavonic) central European population. Different genetic backgrounds or environmental conditions could modify the effect of the analyzed polymorphisms.

\section{Acknowledgment}

This study was supported by project No. NT/12170-5 (Internal Grant Agency, Ministry of Health, Czech Republic).

\section{References}

Bierut LJ, Madden PA, Breslau N, Johnson EO, Hatsukami D, Pomerleau OF, Swan GE, Rutter J, Bertelsen S, Fox L, et al. (2007) Novel genes identified in a high-density genome wide association study for nicotine dependence. Hum Mol Genet 16:24-35.

Bierut LJ, Stitzel JA, Wang JC, Hinrichs AL, Grucza RA, Xuei X, Saccone NL, Saccone SF, Bertelsen S, Fox L, et al. (2008) Variants in nicotinic receptors and risk for nicotine dependence. Am J Psychiatry 165:1163-1171.

Bierut LJ (2009) Nicotine dependence and genetic variation in the nicotinic receptors. Drug Alcohol Depend 104:S64-S69.

Breetvelt EJ, Numans ME, Aukes MF, Hoeben W, Strengman E, Luykx JJ, Bakker SC, Kahn RS, Ophoff RA and Boks MP (2012) The association of the alpha-5 subunit of the nicotinic acetylcholine receptor gene and the brain-derived neurotrophic factor gene with different aspects of smoking behavior. Psychiatr Genet 22:96-98.

Chen LS, Baker TB, Grucza R, Wang J, Johnson EO, Breslau N, Hazukami D, Smith SS, Saccone N, Saccone S, et al. (2012) Dissection of the phenotypic and genotypic associations with nicotinic dependence. Nicotine Tob Res 14:425-433.
Cífková R, Skodová Z, Bruthans J, Adámková V, Jozífová M, Galovcová M, Wohlfahrt P, Krajcoviechová A, Poledne R, Stávek P, et al. (2010) Longitudinal trends in major cardiovascular risk factors in the Czech population between 1985 and 2007/8. Czech MONICA and Czech post-MONICA. Atherosclerosis 211:676-681.

Conlon MS and Bewick MA (2011) Single nucleotide polymorphisms in CHRNA5 rs16969968, CHRNA3 rs578776, and LOC123688 rs8034191 are associated with heaviness of smoking in women in Northeastern Ontario, Canada. Nicotine Tob Res 13:1076-1083.

Day IN, Bolla M, Haddad L, O'Dell S and Humphries SE (1996) Microtiter Array Diagonal Gel Electrophoresis (MADGE) for population scale genotype analyses. Methods Mol Med 5:269-281.

Hartz SM, Short SE, Saccone NL, Culverhouse R, Chen L, Schwantes-An TH, Coon H, Han Y, Stephens SH, Sun J, et al. (2012) Increased genetic vulnerability to smoking at CHRNA5 in early-onset smokers. Arch Gen Psychiatry 69:854-860.

Hubacek JA, Dlouha D, Lanska V and Adamkova V (2012) Lack of an association between three tagging SNPs within the FTO gene and smoking behavior. Nicotine Tob Res 14:998-1002.

Li MD and Burmeister M (2009) New insights into the genetics of addiction. Nat Rev Genet 10:225-231.

Miller SA, Dykes DD AND Polesky HF (1988) A simple salting out procedure for DNA extraction from human nucleated cells. Nucleic Acid Res 16:1215.

Munafò MR, Timpson NJ, David SP, Ebrahim S and Lawlor DA (2009) Association of the DRD2 gene Taq1A polymorphism and smoking behavior: A meta-analysis and new data. Nicotine Tob Res 11:64-76.

Roche AM and Deehan A (2002). Women's alcohol consumption: Emerging patterns, problems and public health implications. Drug Alcohol Rev 21:169-178.

Suriyaprom K, Phonrat B, Chuensumran U, Tungtrongchitr A and Tungtrongchitr R (2012) Association of HTTLPR and 5- $\mathrm{HT}_{2} \mathrm{~A}$ T102C polymorphisms with smoking characteristics and anthropometric profiles of Thai males. Genet Mol Res 11:4360-4369.

Suriyaprom K, Tungtrongchitr R, Thawnashom K and Pimainog Y (2013) BDNF Val66Met polymorphism and serum concentrations of BDNF with smoking in Thai males. Genet Mol Res 12:4925-4933.

Thorgeirsson TE, Gudbjartsson DF, Surakka I, Vink JM, Amin N, Geller F, Sulem P, Rafnar T, Esko T, Walter S, et al. (2010) Sequence variants at CHRNB3-CHRNA6 and CYP2A6 affect smoking behavior. Nat Genet 42:448-453.

Treur JL, Boomsma DI, Lubke GH, Bartels M and Vink JM (2014) The predictive value of smoking expectancy and the heritability of its accuracy. Nicotine Tob Res 16:359-368.

Tunstall-Pedoe H, Kuulasmaa K, Tolonen H, Davidson M and Mendis S (2003) MONICA Monograph and Multimedia Sourcebook 2003. Tunstall-Pedoe H. (editor) World Health Organisation, Geneva, 244 pp.

Vandenbergh DJ, Bennett CJ, Grant MD, Strasser AA, O'Connor R, Stauffer RL, Vogler GP and Kozlowski LT. (2002) Smoking status and the human dopamine transporter variable number of tandem repeats (VNTR) polymorphism: 
Failure to replicate and finding that never-smokers may be different. Nicotine Tob Res 4:333-340.

Verde Z, Santiago C, Rodríguez González-Moro JM, de Lucas Ramos P, López Martín S, Bandrés F, Lucia A and GómezGallego F (2011) 'Smoking genes': A genetic association study. PLoS One 6:e26668.

Ware JJ, van den Bree MB and Munafò MR (2011) Association of the CHRNA5-A3-B4 gene cluster with heaviness of smoking: A meta-analysis. Nicotine Tob Res 3:1167-1175.

Ware JJ, van den Bree M and Munafò MR (2012) From men to mice: CHRNA5/CHRNA3, smoking behavior and disease. Nicotine Tob Res 14:1291-1299.
Zatonski W, Przewozniak K, Sulkowska U, West R and Wojtyla A (2012) Tobacco smoking in countries of the European Union. Ann Agric Environ Med 19:181-192.

\section{Internet Resources}

Court MH (2005-2008) Court-laboratory Hardy-Weinberg calculator, http://www.tufts.edu/ mcourt01/Documents/Court\%201ab\%20-\%20HW\%20calculator.xls (accessed January 2014).

Associate Editor: Maria Rita Passos-Bueno

License information: This is an open-access article distributed under the terms of the Creative Commons Attribution License, which permits unrestricted use, distribution, and reproduction in any medium, provided the original work is properly cited. 